\title{
A Decline in Walking Speed Is Associated With Incident Knee Replacement in Adults With and at Risk for Knee Osteoarthritis
}

\author{
Matthew S. Harkey' (D), Kate L. Lapane ${ }^{2}$ (D), Shao-Hsien Liu² (D), Grace H. Lo3 (D), \\ Timothy E. McAlindon ${ }^{4}$ (i), and Jeffrey B. Driban ${ }^{4}$ (i)
}

\begin{abstract}
Objective. To determine if a 1-year change in walking speed is associated with receiving an incident knee replacement during the following year in adults with and at risk for knee osteoarthritis (OA).

Methods. Using data from the Osteoarthritis Initiative, we determined a 1-year change in the 20-meter walk speed from 3 observation periods (i.e., 0-12, 12-24, and 24-36 months). We operationally defined 1-year change in walking speed as either (1) decline: $\leq-0.1 \mathrm{~m} / \mathrm{s}$ change, $(2)$ no change: between -0.1 and $0.1 \mathrm{~m} / \mathrm{s}$ change, and (3) increase: $\geq 0.1 \mathrm{~m} / \mathrm{s}$ change. Incident knee replacement was defined using each subsequent 1 -year period (i.e., 12-24, 24-36, and 36-48 months). Combining data from the 3 observation periods, we performed a Poisson regression with robust error variance to determine the relative risk between a change in walking speed (exposure) and incident knee replacement over the following year (outcome).

Results. Of the 4264 participants included within this analysis (11,311 total person visits), 115 (3\%) adults received a knee replacement. Decline in walking speed was associated with a $104 \%$ increase in risk [adjusted relative risk (RR) 2.04, 95\% CI 1.40-2.98], while an increase in walking speed associated with a $55 \%$ decrease in risk (RR 0.45 ; $95 \%$ CI $0.22-0.93$ ) of incident knee replacement in the following year compared to a person with no change in walking speed.

Conclusion. A 1-year decline in walking speed is associated with an increased risk, while a 1-year increase in walking speed is associated with a decreased risk of future incident knee replacement.
\end{abstract}

Key Indexing Terms: arthroplasty, gait, knee joint, osteoarthritis

The utilization of knee replacements in the United States is projected to increase by $673 \%$ between 2005 and $2030^{1}$. Knee replacements represent the largest Medicare expenditure for a single procedure, with hospitals being reimbursed $\$ 3.5$ billion in the 2011 fiscal year ${ }^{2}$. Knee osteoarthritis (OA) is one of the most common indications for receiving a knee replacement and more than $50 \%$ of patients with knee OA are expected to receive a knee replacement in their lifetime under the current clinical guidelines $^{3}$. While there are criteria to inform decisions about whether a patient is appropriate for a knee replacement, these criteria only indicate if a knee replacement would be appropriate based on a patient's current status ${ }^{4,5}$. It would be beneficial to also
These analyses were financially supported by a grant from the National Center for Advancing Translational Sciences (TL1-TR001454) and a grant from the National Institute of Arthritis and Musculoskeletal and Skin Diseases of the National Institutes of Health (NIH) under award number R01-AR065977. The Osteoarthritis Initiative (OAI) is a public-private partnership comprising 5 contracts (NO1-AR-2-2258; NO1-AR-2-2259; N01-AR-2-2260; N01-AR-2-2261; N01-AR-2-2262) funded by the NIH, a branch of the Department of Health and Human Services, and conducted by the OAI Study Investigators. Private funding partners include Merck Research Laboratories, Novartis Pharmaceuticals Corporation, GlaxoSmithKline, and Pfizer Inc. Private sector funding for the OAI is managed by the Foundation for the NIH. This manuscript was prepared using an OAI public use dataset and does not necessarily reflect the opinions or views of the OAI investigators, the NIH, or the private funding partners. This work was also supported in part by the Houston Veterans Affairs Health Services Research and Development Center of Excellence (HFP90-020). The views expressed in this article are those of the authors and do not necessarily represent the views of the Department of Veterans Affairs. The funding sources had no role in study design; in the collection, analysis and interpretation of data; in the writing of the report; or in the decision to submit the article for publication.

\begin{abstract}
${ }^{1}$ M.S. Harkey, Postdoctoral Research Fellow, PhD, Department of Population and Quantitative Health Sciences, University of Massachusetts Medical School, Worcester, and Division of Rheumatology, Allergy, and Immunology, Tufts Medical Center, Boston, Massachusetts; ${ }^{2}$ K.L. Lapane, Professor, PhD, S.H. Liu, Assistant Professor, PhD, Department of Population and Quantitative Health Sciences, University of Massachusetts Medical School, Worcester, Massachusetts; ${ }^{3}$ G.H. Lo, Assistant Professor, MD, MSc, Medical Care Line and Research Care Line, Houston Health Services Research and Development Center of Excellence, Michael E. DeBakey VAMC, and Section of Immunology, Allergy, and Rheumatology, Baylor College of Medicine, Houston, Texas; ${ }^{4}$ T.E. McAlindon, Professor, MD, MPH, J.B. Driban, Associate Professor, PhD, Division of Rheumatology, Allergy, and Immunology, Tufts Medical Center, Boston, Massachusetts, USA. The authors declare no conflicts of interest.

Address correspondence to Dr. M. Harkey, Tufts Medical Center, Division of Rheumatology, Allergy, and Immunology, 800 Washington Street, Box 406, Boston, MA 02111,USA.Email:mharkey@tuftsmedicalcenter.org. Full Release Article. For details see Reprints and Permissions at jrheum.org. Accepted for publication June 4, 2020.
\end{abstract}


have a reliable, clinically accessible test to prognostically identify which patients are likely to receive a future knee replacement. This may enable a clinician to start a conversation earlier that includes informing the patient about the need for future knee replacement, encouraging the initiation of and adherence to interventions that prevent/delay the need for knee replacement, or optimizing function prior to surgery to improve postoperative outcomes $^{6,7,8,9,10,11}$.

While imaging outcomes may be prognostic for future knee replacement, they are not readily available to clinicians, therefore limiting their clinical utility compared to more accessible assessments ${ }^{12}$. Habitual walking speed, which can be assessed quickly in a clinic, has been designated as the "6th vital sign," because impaired walking speed may be a general indicator of declining health that predicts a range of important health outcomes: response to rehabilitation, lack of physical activity, functional dependence, frailty, falls, and mortality ${ }^{13}$. Walking speed is also a strong predictor of specific outcomes relevant to knee OA. Adults with slower walking speed have higher odds of developing knee OA over 6 years when compared to faster walkers ${ }^{14}$. Further, adults with late-stage knee OA who elected to get a knee replacement presented with poorer performance on the Timed Up and Go test (i.e., slower walking) 2 years before opting for surgery compared to adults who did not receive a knee replacement ${ }^{15}$. Therefore, walking speed is a clinically accessible measure that is related to OA-related outcomes throughout the disease process, and may serve as an optimal tool to assess the risk of future knee replacement.

Whereas walking speed assessed at a single timepoint is prognostic of knee OA, a longitudinal change in walking speed has been theorized to provide a more sensitive measure of risk assessment ${ }^{16,17}$. For example, when separating a cohort of participants by baseline walking speed, the adults who experienced a fast decline in walking speed were at higher risk of mortality regardless of whether they were considered a slow or fast walker at baseline ${ }^{16}$. Additionally, a 1-year decline in walking speed (i.e., $0.1 \mathrm{~m} / \mathrm{s}$ decline) in adults without knee $\mathrm{OA}$ was associated with an increased risk of developing radiographic knee $\mathrm{OA}$ over 2 years ${ }^{17}$. Decline in walking speed is also associated with worsening preradiographic structural pathology (i.e., increased bone marrow lesion and effusion volume) prior to the development of accelerated knee $\mathrm{OA}^{18}$. Thus, decline in walking speed may be a general indicator of declining health that may associate with increased risk for future knee replacement. However, it is unclear if longitudinal walking speed decline is associated with incident knee replacement in adults with and at risk for knee OA. Conversely, it is unknown if a longitudinal increase in walking speed is associated with a decreased risk of incident knee replacement.

Therefore, the primary purpose of this manuscript was to determine the extent to which a change in walking speed over 1 year was associated with receiving an incident knee replacement within the following year in adults with or at risk for knee OA. Additionally, we performed stratified analyses based on baseline walking speed status (fast walker $\geq 1.22 \mathrm{~m} / \mathrm{s}$; slow walker $<1.22 \mathrm{~m} / \mathrm{s}^{16}$ ) to determine if the association between change in walking speed and incident knee replacement is different among those who started off walking slow or fast. This will help inform clinicians about whether change in walking speed will perform well at predicting knee replacement among everyone regardless of the person's initial walking speed. If walking speed decline is associated with an increased risk of future knee replacement, this may allow for more targeted conservative treatment aimed at improving function and delaying time to knee replacement.

\section{MATERIALS AND METHODS}

Study design and the Osteoarthritis Initiative. We conducted a longitudinal analysis using data from the first 4 years of the Osteoarthritis Initiative (OAI). The OAI is a multicenter (Memorial Hospital of Rhode Island, The Ohio State University, University of Maryland, Johns Hopkins University, and the University of Pittsburgh) cohort study that recruited 4796 adults with or at risk for symptomatic knee OA between February 2004 and May $2006^{19}$. Institutional review boards (IRB) at all of the OAI clinical sites and the OAI coordinating center [University of California, San Francisco (UCSF)] approved the OAI study (approval number 10-00532). The OAI meets all criteria for ethical standards regarding human studies defined in the 1964 Declaration of Helsinki and all amendments made thereafter. Participants provided informed consent prior to participation.

For this study, we created 3 observation periods that each consisted of a 1 -year change in walking speed and the following 1 -year period to determine incidence of knee replacement (Figure 1). Figure 1 demonstrates the exposure (i.e., walking speed change) and outcome (i.e., incident knee replacement) from each of the 3 observation periods. Participants could contribute data in all 3 observation periods depending on the availability of walking speed data and whether they experienced an incident knee replacement over the first 4 years of the OAI. The data from the 3 separate observation periods were combined to determine the overall association between 1-year change in walking speed (exposure) and incident knee replacement during the following year (outcome) throughout the first 4 years of the OAI.

Participant selection. We excluded participants that presented with a knee replacement prior to OAI baseline and those who received a knee replacement between the baseline and 12-month OAI visit. We included OAI participants with 20-meter walking speed data at 2 or more consecutive visits from the OAI baseline to the 36-month follow-up visit. Consecutive visits were needed to calculate 1-year walking speed change in each of the 3 observation periods (i.e., 0-12, 12-24, 24-36 months; Figure 1). Additionally, participants with missing covariate data were excluded.

Walking speed assessment. Walking speed was measured using the 20-meter walk test. Participants performed 2 trials of a timed 20-meter walk at their usual, comfortable walking speed ${ }^{16,17,18,20}$. The participants began each trial in a standing position and an assessor used a stopwatch to start timing when the participant took their first step. An assessor stopped timing when the participant passed a cone positioned 20 meters away. To ensure that participants were not decelerating prior to the end of the test, participants were instructed to maintain their comfortable walking speed for 3 steps past the cone. Testing self-selected walking speed in adults with knee OA has high reliability (intraclass correlation coefficient $>0.93)^{21}$. The time needed to complete the 20 -meters was converted to walking speed $(\mathrm{m} / \mathrm{s})$ and averaged across 2 trials.

One-year change in 20-meter walk speed was calculated as the second year subtracted by the first year in each observation period (i.e., negative values indicate walking speed decline). We operationally defined 1-year change in walking speed as either (1) decline: $\leq-0.1 \mathrm{~m} / \mathrm{s}$ change, (2) no change: between -0.1 and $0.1 \mathrm{~m} / \mathrm{s}$ change, or $(3)$ increase: $\geq 0.1 \mathrm{~m} / \mathrm{s}$ change. We selected a change of $0.1 \mathrm{~m} / \mathrm{s}$ because this amount of change has been previously reported as the minimally clinically important difference for change in walking speed ${ }^{22}$ that is associated with the development of incident radiographic knee $\mathrm{OA}^{17}$, and is associated with worsening structural 


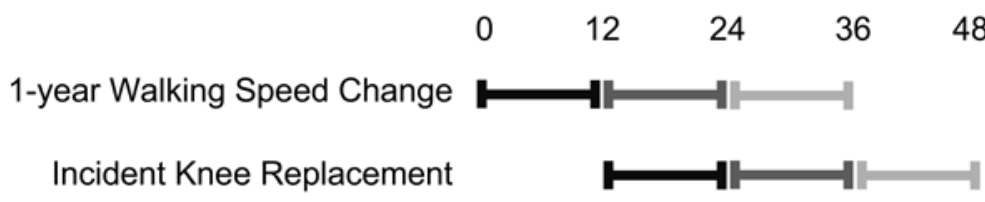

\section{Observation Period $\quad \mathbf{I}=1^{\text {st }} \quad \mathbf{I}=2^{\text {nd }} \quad \|=3^{\text {rd }}$}

Figure 1. Defining the observation periods. An observation period consisted of a 1-year change in walking speed and the following 1-year period to determine incidence of knee replacement. There were 3 observation periods between the baseline and 48-month Osteoarthritis Initiative (OAI) visit. Specifically, in the first observation period, a participant's predictor variable was walking speed change from baseline to 12 -month visit and their outcome was incident knee replacement between the 12 -month visit and the 24-month visit. If a participant received a knee replacement, they were censored from each subsequent period. However, if a participant did not have an incident knee replacement during the first observation period, they could be included in the second observation period with a new predictor (i.e., 12- to 24-month walking speed change) and outcome (i.e., incident knee replacement between the 24-month visit and the 36-month visit).

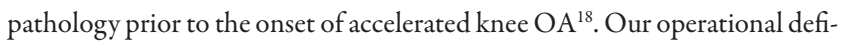
nition of walking speed change exceeds the established smallest detectable difference for decline in 20 -meter walk speed (i.e., $0.07 \mathrm{~m} / \mathrm{s})^{23}$.

Definition of incident knee replacement. A participant was considered to have received an incident knee replacement if a knee replacement (i.e., partial or total; unilateral or bilateral) was self-reported or seen on an OAI study radiograph, and then centrally adjudicated by the OAI Coordinating $\mathrm{Center}^{24}$. Two adjudicators independently reviewed the medical records, and if there was a disagreement, a third physician adjudicator reviewed the medical records. Adults with a knee replacement from 0 to 12 months were removed from the study sample. We then indicated if a person received an incident knee replacement during 1 of the 3 one-year intervals (12-24 months, 24-36 months, 36-48 months). If a participant received a knee replacement, they were censored from each subsequent period.

Operational definition of walking speed status used for stratified analyses. To determine if baseline walking speed serves as an effect modifier of the association between change in walking speed and incident knee replacement, walking speed status was defined separately in each of the 3 observation periods based on a participant's walking speed during the first visit of each observation period (i.e., baseline walking speed). Similar to a previous manuscript associating change in walking speed with mortality in older adults ${ }^{16}$, we stratified the analyses using baseline walking speed as an effect modifier. We operationally defined walking speed status as (1) slow: $<1.22 \mathrm{~m} / \mathrm{s}$, or (2) fast: $\geq 1.22 \mathrm{~m} / \mathrm{s}$. A habitual walking speed of $1.22 \mathrm{~m} / \mathrm{s}$ is a clinically relevant threshold because it is the speed needed to cross a cross-walk during a traffic light change ${ }^{25}$ and represents a minimum performance threshold that indicates the physical ability needed to walk $>6000$ steps per day for adults with and at risk for knee $\mathrm{OA}^{20}$.

Potential confounders. We extracted demographic data at the first visit for all observation periods from the public OAI data files: age, sex (female vs male), BMI $\left(\mathrm{kg} / \mathrm{m}^{2}\right)$, Western Ontario and McMaster Universities Arthritis Index pain (0-20; greater score indicates worse pain), race (White vs non-White), and symptomatic knee OA status (defined by presence of radiographic evidence of knee $\mathrm{OA}=$ centrally read Kellgren-Lawrence grade $\geq 2$ on semiflexed radiographs, AND presence of self-reported frequent pain = evaluated by pain, aching, or stiffness in or around each knee on most days for 1 month within the last year ${ }^{16}$; files: enrollees, version 23 ; allclinical\#\#, version 0.2.2, 1.21, 3.2.1; KXR_SQ_BU, versions 0.6, 1.6, 3.5).

Statistical analysis. We determined the association of a 1-year change in walking speed (i.e., increase, decrease, or no change) and incident knee replacement in the following year over the first 4 years of the OAI with a repeated-measures relative risk estimation by modified Poisson regression with robust error ${ }^{26}$. Since each participant could contribute data from each of the 3 separate observation periods, the modified Poisson regression analysis adjusts for correlations within-person over time, which allows for estimating the relative risk (RR) and CI by using robust error variances ${ }^{27}$. These RR determined how the risk of knee replacement in the increase and decrease in walking speed groups compared to the participants with no change in walking speed. To determine if baseline walking speed serves as an effect modifier of the association between change in walking speed and incident knee replacement, we then conducted a stratified analysis using the same approach to assess this association in slow or fast walkers at the first visit of an observation period.

\section{RESULTS}

The final analysis included 11,311 total observations across 4264 participants ( 3 observations, $\mathrm{n}=3358$; 2 observations, $\mathrm{n}=331$; 1 observation, $n=575$; Figure 2). Between the 12-and 48-month OAI visits, $115(3 \%)$ adults received a knee replacement (i.e., 12-24 month: $\mathrm{n}=33$; 24-36 month: $\mathrm{n}=42$, 36-48 month: $\mathrm{n}=40$ ). Table 1 highlights the demographics for those with an increase, decrease, or no change in walking speed at the initial visit from the first observation period. In the year prior to knee replacement, $37 \%(42 / 115)$ of adults experienced a decline, $7 \%(8 / 115)$ experienced an increase, and 57\% (65/115) experienced no change in walking speed (Table 2 ). In the observations that did not precede a knee replacement, 19\% $(2111 / 11,196)$ experienced a decline, $18 \%(2016 / 11,196)$ experienced an increase, and 63\% $(7069 / 11,196)$ experienced no change in walking speed.

A decline in walking speed over 1 year is associated with a $104 \%$ increase in risk of incident knee replacement in the following year compared to a person with no change (adjusted RR 2.04; 95\% CI 1.40-2.98; Table 2). An increase in walking speed over 1 year is associated with a $55 \%$ decrease in the risk of incident knee replacement in the following year compared to a person with no change in walking speed (RR $0.45 ; 95 \% \mathrm{CI}$ $0.22-0.93$ ). Table 2 also includes the results of the unadjusted analyses that indicate similar associations between walking speed change and knee replacement. 


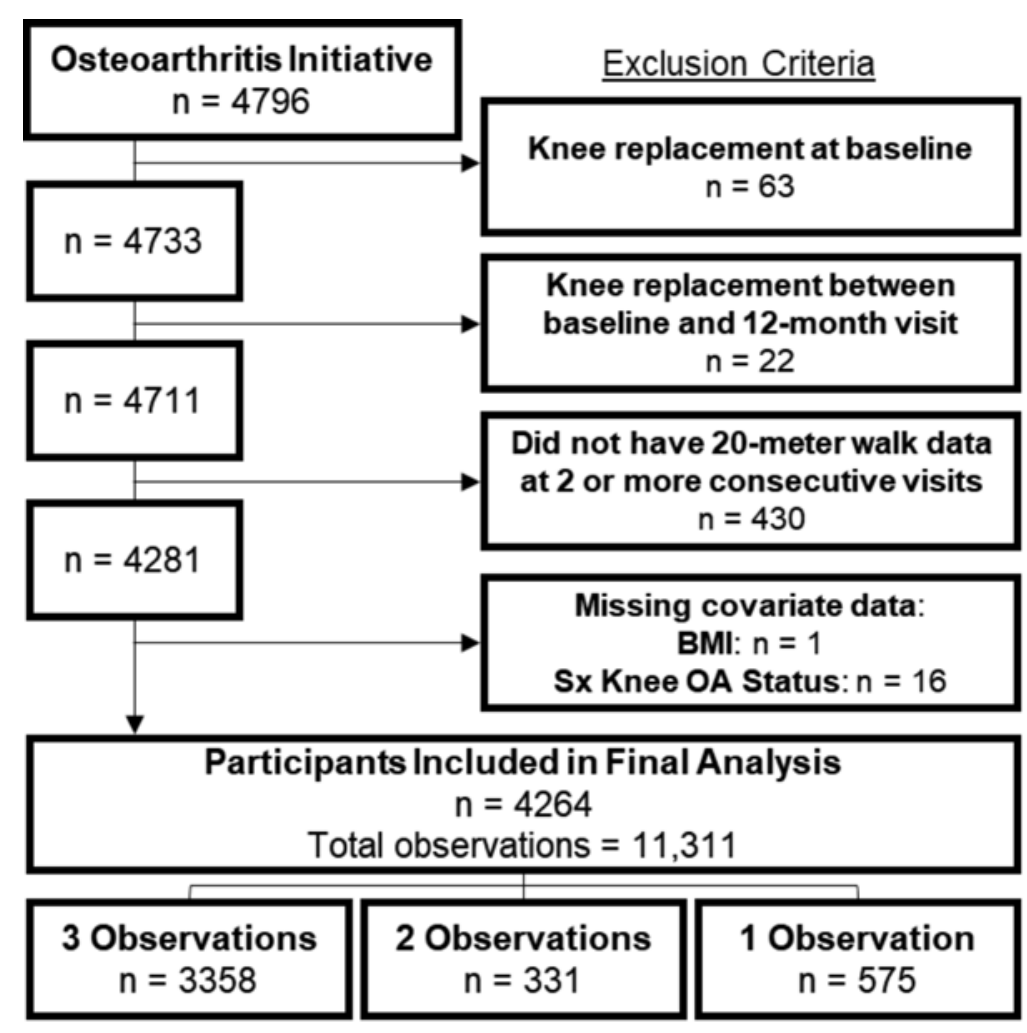

Figure 2. Flow of participant selection. Outlines the exclusion criteria where participants were excluded, and highlights the amount of observations per subject that led to the final participant and observation total included in the final analysis. Sx knee OA status: symptomatic knee osteoarthritis status.

Table 1. Demographics of groups with an increase, decline, or no change in walking speed.

\begin{tabular}{lccc}
\hline & \multicolumn{3}{c}{ 1-year Change in Walking Speed } \\
& No Change & Decline & Increase \\
\hline $\mathrm{n}$ & 2531 & 777 & 956 \\
Age, yrs & $61.4(9.2)$ & $61.4(9.1)$ & $60.3(8.9)$ \\
Female, $\mathrm{n}(\%)$ & $1470(58)$ & $476(61)$ & $515(54)$ \\
Race, White, n (\%) & $2098(83)$ & $609(78)$ & $746(78)$ \\
BMI, kg/m ${ }^{2}$ & $28.5(4.7)$ & $28.6(4.9)$ & $28.4(4.8)$ \\
WOMAC Pain (0-20) & $3.1(3.4)$ & $3.6(3.8)$ & $3.5(3.9)$ \\
Baseline walking speed, $\mathrm{m} / \mathrm{s}$ & $1.33(0.20)$ & $1.43(0.22)$ & $1.24(0.21)$ \\
Symptomatic knee OAc $\mathrm{n}(\%)$ & $757(30)$ & $256(33)$ & $272(28)$ \\
\hline
\end{tabular}

Values are mean $(\mathrm{SD})$ unless otherwise specified. ${ }^{a}$ Walking speed defined as decline: $\leq-0.1 \mathrm{~m} / \mathrm{s}$ change; no change: between -0.1 and $0.1 \mathrm{~m} / \mathrm{s}$ change; or increase: $\geq 0.1 \mathrm{~m} / \mathrm{s}$ change during a participant's first observation period. ${ }^{b}$ Baseline walking speed defined as walking speed at the initial visit of a participant's first observation period. ' Symptomatic knee OA defined by presence of radiographic evidence of knee $\mathrm{OA}=$ centrally read Kellgren-Lawrence grade $\geq 2$ on semiflexed radiographs, AND presence of self-reported frequent pain = evaluated by pain, aching, or stiffness in or around each knee on most days for 1 month within the last year. OA: osteoarthritis; WOMAC: Western Ontario and McMaster Universities Osteoarthritis Index (higher score indicates greater pain).

When stratifying our cohort by baseline walking speed status, a decline in walking speed over 1 year in slow walkers was associated with a $210 \%$ increase in risk of knee replacement in the subsequent year compared to adults with no change (Table 2). Similarly, a decline in walking speed for fast walkers was associated with a $72 \%$ increase in risk of knee replacement in the subsequent year compared to adults with no change in walking speed (Table 2). The RR comparing risk of knee replacement between increase and no change in walking speed for the slow (RR 0.46, 95\% CI 0.17-1.19) and fast (RR 0.42, 95\% CI $0.13-1.35)$ walkers were considered nonsignificant since the 95\% CI crossed 1.0. However, the magnitude of the RR for the slow and fast walkers was similar to the overall group, and the wide CI are likely due to the very small sample size of adults that increased their walking speed and received a knee replacement in the following year.

\section{DISCUSSION}

A decline in walking speed of $0.1 \mathrm{~m} / \mathrm{s}$ over the course of 1 year is associated with a $104 \%$ increase in risk of receiving a knee replacement in the subsequent year when compared to adults that had no change or an increase in their walking speed. Conversely, an increase in walking speed decreased the risk of receiving a knee replacement in the subsequent year by $55 \%$ compared to someone with no change in walking speed. This association between change in walking speed and knee replacement was consistent for adults considered to be slow or fast walkers at their baseline visit of each observation period. These findings highlight that serial monitoring of walking speed is an accessible, clinically feasible test that may be included in a tool 
Table 2. Association between 1-year change in walking speed and incident KR.

\begin{tabular}{|c|c|c|c|c|c|c|c|}
\hline \multirow[t]{3}{*}{ Incident KR } & \multicolumn{3}{|c|}{ 1-year Walking Speed Change } & \multicolumn{4}{|c|}{ RR Between Walking Speed Change Groups } \\
\hline & \multirow{2}{*}{$\begin{array}{c}\text { No Change } \\
\mathrm{n}(\%)\end{array}$} & \multirow{2}{*}{$\begin{array}{c}\text { Decline } \\
\mathrm{n}(\%)\end{array}$} & \multirow{2}{*}{$\begin{array}{c}\text { Increase } \\
\mathrm{n}(\%)\end{array}$} & \multicolumn{2}{|c|}{ Increase vs No Change (Ref) } & \multicolumn{2}{|c|}{ Decline vs No Change (Ref) } \\
\hline & & & & $\mathrm{RR}(95 \% \mathrm{CI})$ & $\operatorname{aRR}(95 \% \mathrm{CI})^{\mathrm{b}}$ & $\mathrm{RR}(95 \% \mathrm{CI})$ & $\operatorname{aRR}(95 \% \mathrm{CI})^{\mathrm{b}}$ \\
\hline No KR (Ref) & $7069(63.1)$ & $2111(18.9)$ & $2016(18.0)$ & 0.43 & 0.45 & 2.15 & 2.04 \\
\hline Incident KR & $65(56.5)$ & $42(36.5)$ & $8(7.0)$ & $(0.21-0.90)$ & $(0.22-0.93)$ & $(1.46-3.16)$ & $(1.40-2.98)$ \\
\hline \multicolumn{8}{|l|}{ Slow walkers ${ }^{c}$} \\
\hline \multicolumn{8}{|l|}{ Fast walkers ${ }^{\mathrm{c}}$} \\
\hline No KR (Ref) & $5139(63.3)$ & $1798(22.2)$ & $1176(14.5)$ & 0.35 & 0.42 & 1.80 & 1.72 \\
\hline Incident KR & $38(58.5)$ & $24(36.9)$ & $3(4.6)$ & $(0.11-1.12)$ & $(0.13-1.35)$ & $(1.08-2.99)$ & $(1.05-2.80)$ \\
\hline
\end{tabular}

Significant values are in bold. ${ }^{a}$ Walking speed change over 1 year defined as no change: between -0.1 and $0.1 \mathrm{~m} / \mathrm{s}$ change, decline: $\leq-0.1 \mathrm{~m} / \mathrm{s}$ change, increase: $\geq 0.1 \mathrm{~m} / \mathrm{s}$ change. Percentage refers to walking speed change within the KR group. ${ }^{\mathrm{b}}$ Adjusted for age, BMI, sex, WOMAC knee pain, symptomatic knee OA, race. ${ }^{c}$ Walking speed status at the first visit in the observation period defined as slow walker: $\leq 1.22 \mathrm{~m} / \mathrm{s}$; fast walker: $>1.22 \mathrm{~m} / \mathrm{s}$. aRR: adjusted relative risk; KR: knee replacement; RR: relative risk; WOMAC: Western Ontario and McMaster Universities Osteoarthritis Index (higher score indicates greater pain).

kit of tests to determine if a patient is at increased or decreased risk for knee replacement within the subsequent year. Improving our ability to identify people at an increased risk of knee replacement may allow for more targeted conservative treatment aimed at improving function and delaying time to knee replacement ${ }^{7}$.

The results of our stratified analysis based on baseline walking speed status are in agreement with previous studies to highlight the importance of assessing change in walking speed, and not just baseline walking speed, as a sensitive marker for future poor outcomes ${ }^{16,17}$. A previous study using a similar baseline walking speed stratification found that regardless of whether a person started as a slow or fast walker, adults within both groups that exhibited the fastest trajectory of walking speed decline were at greater risk for mortality within 8 years $^{16}$. Similarly, we found that baseline walking speed is not an effect modifier of the association between walking speed change and knee replacement, because a decline in walking speed is associated with future knee replacement regardless of being a fast or slow walker at baseline. This indicates that even if a person is considered to have a "functional" baseline walking speed (i.e., $\geq 1.22 \mathrm{~m} / \mathrm{s}$ ) 25 , a 1 -year decline in walking speed provides further insight into their health status and is associated with an increased risk of future knee replacement. While assessing walking speed at a single timepoint is an important indicator for future negative health outcomes ${ }^{13}$, the sensitivity of a single baseline assessment of walking speed may be limited because it does not provide any indication of that individual's history and how their speed is changing over time. In addition to the negative implications of a decline in walking speed, our results also indicate that an increase in 1-year walking speed is associated with a decreased risk of knee replacement in the subsequent year compared to adults with no change in walking speed. Therefore, serial walking speed assessments may provide additional information to walking speed at a discrete timepoint, as this may help identify patients on a trajectory of walking speed decline prior to the occurrence of future adverse events.

This study highlights that walking speed change is associated with an incident knee replacement during the subsequent year in adults with and at risk for knee OA, but there are some limitations that need to be taken into consideration. Walking speed change was assessed over a 12-month period collected at 2 distinct timepoints and may not reflect the full extent of walking speed fluctuations that may occur throughout an entire year. However, this timing of the assessments may be easily translatable into a clinician's assessment at annual follow-up visits. Since calculation of walking speed change requires 2 consecutive visits, we may have excluded participants with the greatest amount of physical limitations who were unable to complete walking speed assessments over 2 consecutive visits. However, since the design of our study includes 3 different observation periods, we were able to maximize the inclusion of participants throughout the first 4 years of the OAI. Due to the inclusion criteria of the OAI, the results of this study should only be generalized to adults between the ages of 45-79 with knee OA or those matching age-specific criteria used by the OAI to identify adults at risk for knee $\mathrm{OA}^{19}$. While a decline in walking speed does increase the risk of a person receiving an incident knee replacement within the following year, the overall percentage of adults presenting with a decline in walking speed and receiving a knee replacement is small (Table 2). Since electing to undergo a knee replacement is influenced by many extraneous factors (e.g., race, geographic area, healthcare coverage), serial walking speed testing cannot serve as a singular test to estimate a person's risk for future knee replacement but may be included in a larger tool kit to help accurately estimate a patient's risk of future knee replacement. Our results indicate that people with declining walking speed are more likely to receive a knee replacement compared to those with no change or an increase in walking speed, but our analyses cannot determine if declining walking speed is an indication of appropriateness for knee replacement. Future studies are needed to determine if adding in a serial walking speed test can improve current classification systems for determining the appropriateness of knee replacement ${ }^{4}$. While our results indicate an association with knee replacement between both an increase and a 
decrease in walking speed, future studies are needed to determine if there is a causal relationship between interventions that create an increase in walking speed and preventing or delaying the time to knee replacement. The overall incidence of knee replacement in our study was low, with only 115 out of 4264 participants over our 3 observation periods. While the incidence of this procedure may be low, knee replacements have significant ramifications on individual patients and the healthcare system, and being able to determine who is at risk is the first step toward more targeted interventions.

In conclusion, a 1-year decline in walking speed of $0.1 \mathrm{~m} / \mathrm{s}$ is associated with a $104 \%$ increase in risk of knee replacement within the following year compared to people with no change in walking speed. Additionally, an increase in walking speed decreased the risk of receiving a knee replacement in the subsequent year by $55 \%$ compared to someone with no change in walking speed. Walking speed decline represents a clinically accessible outcome that can easily be monitored annually to help identify adults at an increased risk of future knee replacement. Further studies are needed to determine if interventions that help prevent a decline or create an increase in walking speed will help to prolong the time to knee replacement.

\section{REFERENCES}

1. Kurtz S, Ong K, Lau E, Mowat F, Halpern M. Projections of primary and revision hip and knee arthroplasty in the United States from 2005 to 2030. J Bone Joint Surg Am 2007;89:780-5.

2. Culler SD, Jevsevar DS, Shea KG, Wright KK, Simon AW. The incremental hospital cost and length-of-stay associated with treating adverse events among Medicare beneficiaries undergoing TKA. J Arthroplasty 2015;30:19-25.

3. Losina E, Paltiel AD, Weinstein AM, Yelin E, Hunter DJ, Chen SP, et al. Lifetime medical costs of knee osteoarthritis management in the United States: impact of extending indications for total knee arthroplasty. Arthritis Care Res 2015;67:203-15.

4. Escobar-Martinez A, Perera RA, Riddle DL. Development and underlying structure of a second-generation appropriateness classification system for total knee arthroplasty. Arthritis Care Res 2020 Feb 26 (E-pub ahead of print).

5. Escobar A, Quintana JM, Aróstegui I, Azkárate J, Güenaga JI, Arenaza JC, et al. Development of explicit criteria for total knee replacement. Int J Technol Assess Health Care 2003;19:57-70.

6. Arden N, Richette P, Cooper C, Bruyère O, Abadie E, Branco $\mathrm{J}$, et al. Can we identify patients with high risk of osteoarthritis progression who will respond to treatment? A focus on biomarkers and frailty. Drugs Aging 2015;32:525-35.

7. Skou ST, Roos EM, Laursen MB, Rathleff MS, Arendt-Nielsen L, Rasmussen S, et al. Total knee replacement and non-surgical treatment of knee osteoarthritis: 2-year outcome from two parallel randomized controlled trials. Osteoarthritis Cartilage 2018;26:1170-80.

8. Teoh LS, Eyles JP, Makovey J, Williams M, Kwoh CK, Hunter DJ. Observational study of the impact of an individualized multidisciplinary chronic care program for hip and knee osteoarthritis treatment on willingness for surgery. Int $\mathrm{J}$ Rheum Dis 2016;20:1383-92.

9. Yu D, Jordan KP, Snell KIE, Riley RD, Bedson J, Edwards JJ, et al. Development and validation of prediction models to estimate risk of primary total hip and knee replacements using data from the UK: two prospective open cohorts using the UK clinical practice research datalink. Ann Rheum Dis 2018;78:91-9.
10. Skoffer B, Maribo T, Mechlenburg I, Hansen PM, Søballe K, Dalgas U. Efficacy of preoperative progressive resistance training on postoperative outcomes in patients undergoing total knee arthroplasty. Arthritis Care Res 2016;68:1239-51.

11. Calatayud J, Casaña J, Ezzatvar Y, Jakobsen MD, Sundstrup E, Andersen LL. High-intensity preoperative training improves physical and functional recovery in the early post-operative periods after total knee arthroplasty: a randomized controlled trial. Knee Surg Sports Traumatol Arthrosc 2016;25:2864-72.

12. Kwoh CK, Guerhing H, Aydemir A, Hannon MJ, Eckstein F, Hochberg MC. Predicting knee replacement in participants eligible for disease-modifying osteoarthritis drug treatment with structural endpoints. Osteoarthritis Cartilage 2020;28:782-91.

13. Fritz S, Lusardi M. White paper: "Walking speed: The sixth vital sign”. J Geriatr Phys Ther 2009;32:46-9.

14. Purser JL, Golightly YM, Feng Q, Helmick CG, Renner JB, Jordan JM. Association of slower walking speed with incident knee osteoarthritis-related outcomes. Arthritis Care Res 2012;64:1028-35.

15. Zeni JA Jr, Axe MJ, Snyder-Mackler L. Clinical predictors of elective total joint replacement in persons with end-stage knee osteoarthritis. BMC Musculoskelet Disord 2010;11:86.

16. White DK, Neogi T, Nevitt MC, Peloquin CE, Zhu Y, Boudreau $\mathrm{RM}$, et al. Trajectories of gait speed predict mortality in well-functioning older adults: the health, aging and body composition study. J Gerontol A Biol Sci Med Sci 2013;68:456-64.

17. Herzog MM, Driban JB, Cattano NM, Cameron KL, Tourville TW, Marshall SW, et al. Risk of knee osteoarthritis over 24 months in individuals who decrease walking speed during a 12-month period: data from the Osteoarthritis Initiative. J Rheumatol 2017;44:1265-70.

18. Harkey MS, Price L, McAlindon TE, Davis JE, Stout AC, Lu B, et al. Association between declining walking speed and increasing bone marrow lesion and effusion volume in individuals with accelerated knee osteoarthritis. Arthritis Care Res 2019;71:259-70.

19. The Osteoarthritis Initiative. [Internet. Accessed November 18, 2020.] Available from: data-archive.nimh.nih.gov/oai

20. Master H, Thoma LM, Christiansen MB, Polakowski E, Schmitt LA, White DK. Minimum performance on clinical tests of physical function to predict walking 6,000 steps/day in knee osteoarthritis: an observational study. Arthritis Care Res 2018;70:1005-11.

21. Fransen M, Crosbie J, Edmonds J. Reliability of gait measurements in people with osteoarthritis of the knee. Phys Ther 1997;77:944-53.

22. Bohannon RW, Glenney SS. Minimal clinically important difference for change in comfortable gait speed of adults with pathology: a systematic review. J Eval Clin Pract 2014;20:295-300.

23. Motyl JM, Driban JB, McAdams E, Price L, McAlindon TE. Test-retest reliability and sensitivity of the 20 -meter walk test among patients with knee osteoarthritis. BMC Musculoskelet Disord 2013;14:166.

24. Davis JE, Liu SH, Lapane K, Harkey MS, Price LL, Lu B, et al. Adults with incident accelerated knee osteoarthritis are more likely to receive a knee replacement: data from the Osteoarthritis Initiative. Clin Rheumatol 2018;37:1115-8.

25. Langlois JA, Keyl PM, Guralnik JM, Foley DJ, Marottoli RA, Wallace RB. Characteristics of older pedestrians who have difficulty crossing the street. Am J Public Health 1997;87:393-7.

26. Zou G. A modified poisson regression approach to prospective studies with binary data. Am J Epidemiol 2004;159:702-6.

27. Yelland LN, Salter AB, Ryan P. Performance of the modified Poisson regression approach for estimating relative risks from clustered prospective data. Am J Epidemiol 2011;174:984-92. 